\title{
Dissipation effects in spin-Hall transport of electrons and holes
}

\author{
John Schliemann and Daniel Loss \\ Department of Physics and Astronomy, University of Basel, CH-4056 Basel, Switzerland
}

(Received 21 January 2004; published 21 April 2004)

\begin{abstract}
We investigate the spin-Hall effect of both electrons and holes in semiconductors using the Kubo formula in the correct zero-frequency limit taking into account the finite momentum relaxation time of carriers in real semiconductors. This approach allows us to analyze the range of validity of recent theoretical findings. In particular, the spin-Hall conductivity vanishes for vanishing spin-orbit coupling if the correct zero-frequency limit is performed.
\end{abstract}

DOI: 10.1103/PhysRevB.69.165315

PACS number(s): 73.63.-b, 85.75.-d

\section{INTRODUCTION}

In the recent years, an increasing interest in spindependent phenomena in semiconductors has developed, mostly in the field of spin electronics, which has by now become a major branch of solid-state research. ${ }^{1-3}$ One of the most investigated issues in this field is the influence of spinorbit coupling on various transport properties of both electrons and holes. Many of these studies were inspired by the paradigmatic proposal of a spin field-effect transistor due to Datta and Das; ${ }^{4}$ for recent work in this direction see, e.g., Refs. 5-13. Most recently, interesting theoretical studies on the spin-Hall effect have been performed. ${ }^{14-16}$ This effect amounts in a spin current (as opposed to a charge current) driven by an electric field perpendicular to it. In the present paper we reexamine these findings using the Kubo formula with full frequency dependence, treating both the case of electrons ${ }^{15}$ and holes, ${ }^{14,16}$ and analyze the range of validity of previous theoretical results obtained for the case of direct current. Here it is crucial to perform the correct zerofrequency limit taking into account an imaginary part of the frequencies occurring the Kubo formula.

The notion of the spin-Hall effect in systems of itinerant spinful carriers was considered first by Dyakonov and Perel ${ }^{17}$ in the early seventies, and, independently, in a more recent paper by Hirsch. ${ }^{18}$ In these studies the predicted spin-Hall effect is due to spin-orbit effects influencing scattering processes upon static impurities. Following the terminology used in ${ }^{15,16}$ this is referred to as the extrinsic spin-Hall effect since it depends on impurity scattering. This is in contrast to the intrinsic spin-Hall effect predicted very recently in Refs. 14-16 which is entirely due to spin-orbit coupling terms in the single-particle carrier Hamiltonian and independent of any scattering process. As we shall see below, this distinction between intrinsic and extrinsic effects becomes ambiguous in the limit of weak spin-orbit coupling when lifetime effects of carrier quasiparticles have to be taken into account.

Yet another type of spin-Hall effect was studied recently by Meier and Loss ${ }^{19}$ in a two-dimensional Heisenberg model consisting of isolated spins, in contrast to the itinerant-carrier systems mentioned before.

Spin-orbit coupling also induces off-diagonal components of the conductivity tensor for charge transport. An important example is the anomalous Hall effect as it occurs in semiconductors in the presence of magnetic impurities. ${ }^{20}$ Here, as in the case of the aforementioned spin-Hall effect, the offdiagonal elements of the charge conductivity tensor are the same in magnitude but differ in sign. Therefore, this antisymmetric conductivity tensor has the same components in all orthogonal coordinate systems, and in this sense the transport properties are isotropic. This is different from charge transport of electrons in quantum wells as investigated recently, Ref. 12. In such systems, the presence of spin-orbit coupling of both the Rashba ${ }^{21}$ and the Dresselhaus ${ }^{22}$ type leads to anisotropic dispersion relations and Fermi contours. This feature leads to symmetric off-diagonal elements in the conductivity tensor and therefore to preferred eigendirections for charge transport. ${ }^{11,12}$ This predicted effect offers a possibility to detect spin-orbit coupling by measuring diffusive spinunpolarized charge currents in a Hall-type geometry, which should be a comparatively simple experimental task.

This paper is organized as follows. In Sec. II we summarize elementary properties of linear-response theory as given by the Kubo formula. This technique is applied then in Sec. III to spin-Hall transport of electrons in a quantum well in the presence of Rashba spin-orbit coupling. In Sec. IV we investigate the case of bulk holes described by the Luttinger Hamiltonian in the spherical approximation. We end with conclusions in Sec. V.

\section{KUBO FORMULA AND ZERO-FREQUENCY LIMIT}

Our present study of spin-Hall effect of electrons and holes in semiconductors is based on the usual Kubo formula with full frequency dependence for a spatially homogeneous electric field, ${ }^{23}$

$$
\begin{aligned}
\sigma_{x y}^{S, z}(\omega)= & \frac{e}{A(\omega+i \eta)} \int_{0}^{\infty} e^{i(\omega+i \eta) t} \\
& \times \sum_{\vec{k}, \mu} f\left(\varepsilon_{\mu}(\vec{k})\right)\left\langle\vec{k}, \mu\left|\left[j_{x}^{S, z}(t), v_{y}(0)\right]\right| \vec{k}, \mu\right\rangle,
\end{aligned}
$$

where we have assumed zero temperature $T=0$ and noninteracting carriers, which allows to formulate the two-body Green's function entering the conductivity Kubo formula in terms of single-particle operators. $A$ is the volume of the system, $e$ is the elementary charge, and $f\left(\varepsilon_{\mu}(\vec{k})\right)$ is the $T$ 
$=0$ Fermi distribution function for energy $\varepsilon_{\mu}(\vec{k})$ at wave vector $\vec{k}$ in a dispersion branch labeled by $\mu$. The velocity operators are given by $\vec{v}=i[\mathcal{H}, \vec{r}] / \hbar$ where $\vec{r}$ is the position operator, and $\mathcal{H}$ is the single-particle Hamiltonian not including the external electric field. The spin-current operator (in the Dirac picture) for spin moment polarized along the $z$ direction and flowing in the $x$ direction is given by

$$
j_{x}^{S, z}(t)=e^{i \mathcal{H} t / \hbar} \frac{1}{2}\left(s^{z} v_{x}+v_{x} s^{z}\right) e^{-i \mathcal{H} t / \hbar},
$$

where $\vec{s}$ is the spin operator. The right-hand side of Eq. (1) has to be understood in the limit of vanishing imaginary part $\eta>0$ in the frequency argument. This imaginary part in the frequency reflects the fact that the external electric field is assumed to be switched on adiabatically starting from the infinite past of the system, and it also ensures causality properties of the retarded Green's function occurring in Eq. (1). In general, and as we will discuss in more detail below, the limiting process $\eta \rightarrow 0$ does not commute with other limits, and, in particular, the dc limit $\omega \rightarrow 0$ has to be taken with care. ${ }^{23}$ In the presence of random impurity scattering, the retarded two-body Green's function in Eq. (1) will generically have a frequency argument with positive imaginary part. ${ }^{23}$ In this case the limit $\eta \rightarrow 0$ is unproblematic, and the imaginary part of the frequency argument is just due to impurity scattering and/or other (many-body) effects. Generically, and as we will discuss in more detail below, the imaginary part $\eta>0$ corresponds to a finite carrier quasiparticle lifetime.

\section{ELECTRONS WITH RASHBA COUPLING}

Sinova et al. ${ }^{15}$ have considered the spin-Hall effect of noninteracting electrons confined to the two-dimensional (xy) plane of a quantum well and being subject to Rashba spin-orbit coupling, ${ }^{3,21}$

$$
\mathcal{H}=\frac{\vec{p}^{2}}{2 m^{*}}+\frac{\alpha}{\hbar}\left(p_{x} \sigma^{y}-p_{y} \sigma^{x}\right),
$$

where $m^{*}$ is the effective mass, $\alpha$ the Rashba coefficient, and the other notations are standard. We note that in systems where both the Rashba and the Dresselhaus spin-orbit coupling ${ }^{3,22}$ are present, various interesting transport effects can arise from the interplay of these two terms, for recent studies see, e.g., Refs. 5, 7-9, 11, 12. For simplicity, however, we shall concentrate here on the Rashba term only. The Hamiltonian (3) has two energy branches,

$$
\varepsilon_{ \pm}(\vec{k})=\frac{\hbar^{2} k^{2}}{2 m^{*}} \pm \alpha k
$$

with eigenstates

$$
\langle\vec{r} \mid \vec{k}, \pm\rangle=\frac{e^{i \vec{k} r}}{\sqrt{A}} \frac{1}{\sqrt{2}}\left(\begin{array}{c}
1 \\
\pm e^{i \chi(\vec{k})}
\end{array}\right),
$$

where $\chi(\vec{k})=\arg \left(-k_{y}+i k_{x}\right)$. By a straightforward calculation one obtains for the spin-Hall conductivity

$$
\begin{aligned}
\sigma_{x y}^{S, z}(\omega) & =-\sigma_{y x}^{S, z}(\omega) \\
& =-\frac{e}{4 \pi} \frac{\alpha}{m^{*}} \int_{k_{f}^{+}}^{k_{f}^{-}} d k \frac{k^{2}}{(\omega+i \eta)^{2}-\left(\frac{2 \alpha k}{\hbar}\right)^{2}},
\end{aligned}
$$

where

$$
k_{f}^{ \pm}=\sqrt{\frac{2 m^{*}}{\hbar^{2}} \varepsilon_{f}+\left(\frac{m^{*}}{\hbar^{2}}\right)^{2} \alpha^{2}} \mp \frac{m^{*}}{\hbar^{2}} \alpha
$$

are the Fermi momenta on the two dispersion branches for positive Fermi energy $\varepsilon_{f}>0$. In the presence of scattering on static random impurities, the imaginary part $\eta>0$ in the frequency argument is given, to lowest order in the Rashba coefficient and the impurity potential, by the inverse of the momentum relaxation time. This is certainly a very intuitive result; however, let us sketch a formal proof for this assertion. The time-dependent spin-current operator in the presence of Rashba coupling reads

$$
j_{x}^{S, z}(t)=\frac{\hbar}{2 m^{*}} \sigma^{z}(t) p_{x}(t),
$$

where the time evolution includes impurity scattering. To lowest order in the spin-orbit coupling and the impurity scattering we have

$$
j_{x}^{S, z}(t) \approx \frac{\hbar}{2 m^{*}} \sigma_{0}^{z}(t) p_{x}^{0}(t),
$$

where the time evolution of $\sigma_{0}^{z}$ is only due to the Hamiltonian (3) and evaluated in the above expression (6), while $p_{x}^{0}(t)$ contains the impurity scattering but not the spin-orbit coupling. Now it is useful to note that, in order to compute the expectation values in the Kubo formula Eq. (1), only matrix elements of the time-dependent momentum operator $p_{x}^{0}(t)$ which are diagonal in the wave vector index are needed. This enables to apply superoperator techniques developed in Ref. 24 yielding

$$
\left[p_{x}^{0}(t)\right]_{\vec{k} \vec{k}} \approx\left[e^{-\Omega_{0} t} p_{x}^{0}(0)\right]_{\vec{k} \vec{k}}=\left[e^{-t / \tau} p_{x}^{0}(0)\right]_{\vec{k} \vec{k}},
$$

where $\Omega_{0}$ is the scattering master operator in lowest order of the scattering potential. ${ }^{24}$ It is the same operator as it occurs as the scattering term in the usual Boltzmann equation when evaluated in lowest oder via Fermi's golden rule. For impurity potentials being isotropic in real space, the momentum $p_{x}$ is an exact eigenfunction of $\Omega_{0}$, and the eigenvalue is given by the well-known inverse momentum relaxation time $1 / \tau(\varepsilon)$ Refs. 24 and 25 which in general depends on the energy $\varepsilon(\vec{k})$. To lowest order in the Rashba coupling, this energy argument can be replaced with the Fermi energy in the absence of spin-orbit interaction. We note that this momentum relaxation rate $1 / \tau$ is the same as obtained in the standard diagrammatic approach and thus contains the vertex correction. ${ }^{23}$ However, this vertex correction vanishes for short-range isotropic scatterers. The above argumentation refers to the Rashba Hamiltonian (3) for conduction-band elec- 
trons; similar considerations can be performed in the case of valence-band holes to be discussed further below.

For $\omega=0$, but finite momentum relaxation rate $1 / \tau>0$, Eq. (6) yields

$$
\begin{aligned}
\sigma_{x y}^{S, z}(0) & =-\sigma_{y x}^{S, z}(0) \\
& =\frac{e}{8 \pi}-\frac{e}{32 \pi} \frac{\hbar / \tau}{\varepsilon_{R}} \cdot \tan ^{-1}\left[4 \frac{\varepsilon_{R}}{\hbar / \tau}\left(1+8 \frac{\varepsilon_{R} \varepsilon_{f}}{(\hbar / \tau)^{2}}\right)^{-1}\right],
\end{aligned}
$$

where we have introduced the "Rashba energy" $\varepsilon_{R}$ $=m^{*} \alpha^{2} / \hbar^{2}$. Clearly, this is the energy which has to be compared with the energy scale $\hbar / \tau$ of the impurity scattering in order to obtain the correct zero-frequency limit of the spinHall conductivity. If the impurity scattering is weak compared to spin-orbit coupling, $\varepsilon_{R} \tau / \hbar \gg 1$, we have the expansion

$$
\sigma_{x y}^{S, z}(0)=\frac{e}{8 \pi}-\frac{e}{64 \pi} \frac{(\hbar / \tau)^{2}}{\varepsilon_{R} \varepsilon_{f}}+O\left[\left(\frac{\hbar / \tau}{\varepsilon_{R}}\right)^{2}\right],
$$

where we have additionally assumed that the Fermi energy $\varepsilon_{f}$ is larger or at least of the same order as $\varepsilon_{R}$, which is usually the case in experimental situations. The zeroth-order contribution $e / 8 \pi$ is the result obtained by Sinova et al. using directly a zero-frequency perturbative expression for the spin-Hall current neglecting effects of a finite electron quasiparticle lifetime, [cf. Eq. (8) in Ref. 15]. Remarkably, this value is universal in the sense that it is independent of $\alpha$. Therefore, it predicts a finite spin-Hall conductivity even in the limit of vanishing spin-orbit coupling, $\alpha \rightarrow 0$, which is certainly an unphysical feature. However, this paradox can be resolved by the observation that the above two limiting processes do not commute. In fact, in the opposite limit $\varepsilon_{R} \tau / \hbar \ll 1$ the lowest order of the second term on the rhs of Eq. (6) cancels the first one, and the spin-Hall conductivity is given in leading order by

$$
\sigma_{x y}^{S, z}(0)=\frac{e}{\pi} \frac{\varepsilon_{R} \varepsilon_{f}}{(\hbar / \tau)^{2}}+O\left[\left(\frac{\varepsilon_{R}}{\hbar / \tau}\right)^{2}\right] .
$$

Thus, to obtain the correct dc spin-Hall conductivity, the "Rashba energy" $\varepsilon_{R}=m^{*} \alpha^{2} / \hbar^{2}$ should be compared with the energy scale $\hbar / \tau$ of the impurity scattering. If $\varepsilon_{R} \gg \hbar / \tau$ the spin-Hall conductivity is close to its "universal" value $e / 8 \pi$, while it vanishes for small spin-orbit coupling and finite impurity scattering. In epitaxially grown GaAs quantum wells mobilities $\mu=e \tau / m^{*}$ of order $100 \mathrm{~m}^{2} / \mathrm{Vs}$ can routinely be achieved, corresponding to values for $\hbar / \tau$ of order $0.01 \mathrm{meV}$. This is safely smaller than typical values for the Rashba energy reported from experiments ${ }^{26-34}$ being of order $0.1, \ldots, 1.0 \mathrm{meV}$. However, it should be noted that the Rashba coefficient is typically proportional to an external electric field applied in the growth direction of the quantum well. Therefore, also smaller values of the Rashba energy are possible where the finite momentum relaxation time will influence the value of the spin-Hall conductivity.

\section{HOLES IN THE VALENCE BAND OF III-V SEMICONDUCTORS}

Murakami, Nagaosa, and Zhang have investigated spinHall transport in three-dimensional bulk systems of holes in the valence band of III-V semiconductors. ${ }^{14}$ These authors used a phenomenological semiclassical theory to describe adiabatic hole dynamics. Their work was revisited most recently by Culcer et al. ${ }^{16}$ within the framework of a semiclassical theory of wave packet dynamics. Here we will evaluate the spin-Hall conductivity using the rigorous Kubo formula (1). Our starting point ${ }^{14,16}$ is Luttinger's four-band Hamiltonian for heavy and light holes in the spherical approximation, ${ }^{35}$

$$
\mathcal{H}=\frac{1}{2 m}\left[\left(\gamma_{1}+\frac{5}{2} \gamma_{2}\right) \vec{p}^{2}-2 \gamma_{2}(\vec{p} \cdot \vec{S})^{2}\right] .
$$

Here $m$ is the bare electron mass, and $\vec{S}$ are spin$3 / 2$-operators. The dimensionless Luttinger parameter $\gamma_{1}$ and $\gamma_{2}$ describe the valence band of the specific material with effects of spin-orbit coupling being included in $\gamma_{2}$. The eigenstates of Eq. (14) can be chosen to be eigenstates of the helicity operator $\lambda=(\vec{k} \cdot \vec{S}) / k$. The heavy holes correspond to $\lambda= \pm 3 / 2$, while the light holes have $\lambda= \pm 1 / 2$. From the Kubo formula (1) one finds for the frequency-dependent spin-Hall conductivity after lengthy but elementary calculations

$$
\begin{aligned}
\sigma_{x y}^{S, z}(\omega)= & -\frac{e}{\pi^{2}}\left(\frac{\hbar}{m}\right)^{2}\left(\gamma_{1}+2 \gamma_{2}\right) \gamma_{2} \cdot \int_{k_{f}^{l}}^{k_{f}^{h}} d k \\
& \times \frac{k^{4}}{(\omega+i / \tau)^{2}-\left(\frac{2 \hbar}{m} \gamma_{2} k^{2}\right)^{2}},
\end{aligned}
$$

where

$$
k_{f}^{h / l}=\sqrt{\frac{2 m}{\hbar^{2}} \varepsilon_{f} \frac{1}{\gamma_{1} \mp 2 \gamma_{2}}}
$$

are the Fermi wave numbers for heavy and light holes, respectively. Again it is instructive to consider the case for weak spin-orbit coupling, $\gamma_{2} \ll \gamma_{1}$. For $\gamma_{2}=0$ we have $k_{f}^{h}$ $=k_{f}^{l}=: k_{F}^{0}=\sqrt{2 m \varepsilon_{f} / \gamma_{1} \hbar^{2}}$, and therefore the integral in Eq. (15) vanishes for finite $1 / \tau>0$ and all frequencies $\omega$. Thus, as before for the case of electrons, the dc spin-Hall conductivity vanishes for vanishing spin-orbit coupling if a finite momentum relaxation rate is taken into account. This result is in contrast to statements in Refs. 14 and 16, where such dissipation effects were neglected. Specifically, for $\omega=0$ we have 


$$
\begin{aligned}
\sigma_{x y}^{S, z}(0)= & \frac{e}{4 \pi^{2}} \frac{\gamma_{1}+2 \gamma_{2}}{\gamma_{2}} \cdot\left(k_{f}^{h}-k_{f}^{l}\right. \\
& \left.-\int_{k_{f}^{l}}^{k_{f}^{h}} d k \frac{1}{1+\left(\frac{2}{\hbar / \tau} \frac{\hbar^{2}}{m} \gamma_{2} k^{2}\right)^{2}}\right) .
\end{aligned}
$$

The remaining integral is elementary leading to a rather tedious expression which shall not be given here. However, we see that the value of the above integral is governed by the ratio of $\hbar / \tau$ and the "spin-orbit energy" $\varepsilon_{s o}:=\hbar^{2} \gamma_{2}\left(k_{f}^{0}\right)^{2} / m$ $=2 \varepsilon_{f} \gamma_{2} / \gamma_{1}$, since $k_{f}^{0}$ is a typical wave number in the integration interval. If $\hbar / \tau \gg \varepsilon_{s o}$ the spin-Hall conductivity vanishes as

$$
\sigma_{x y}^{S, z}(0)=\frac{e}{\pi^{2}} 4 k_{f}^{0}\left(\frac{\varepsilon_{s o}}{\hbar / \tau}\right)^{2} \frac{\gamma_{2}}{\gamma_{1}}+O\left[\left(\frac{\varepsilon_{s o}}{\hbar / \tau}\right)^{4},\left(\frac{\varepsilon_{s o}}{\hbar / \tau}\right)^{2}\left(\frac{\gamma_{2}}{\gamma_{1}}\right)^{2}\right],
$$

where we have also assumed that the ratio $\gamma_{2} / \gamma_{1}$ is small as it is usually the case. ${ }^{36}$ In the opposite case $\hbar / \tau \ll \varepsilon_{\text {so }}$ one finds

$$
\begin{aligned}
\sigma_{x y}^{S, z}(0)= & \frac{e}{4 \pi^{2}} \frac{\gamma_{1}+2 \gamma_{2}}{\gamma_{2}}\left\{k_{f}^{h}-k_{f}^{l}+\frac{\left(k_{f}^{0}\right)^{4}}{12}\left[\left(\frac{1}{k_{f}^{h}}\right)^{3}-\left(\frac{1}{k_{f}^{l}}\right)^{3}\right]\right. \\
& \left.\times\left(\frac{\hbar / \tau}{\varepsilon_{\text {so }}}\right)^{2}\right\}+O\left[\left(\frac{\hbar / \tau}{\varepsilon_{\text {so }}}\right)^{4}\right] .
\end{aligned}
$$

We note that the zeroth order of this result agrees with the expression given in Ref. 16 for the dc spin-Hall conductivity neglecting dissipation effects, ${ }^{37}$ but differs from the result reported in Ref. 14. On the present stage we cannot comment on the question whether this difference is an artifact of the semiclassical approach used in Ref. 14, whereas the present result is obtained from a rigorous linear-response theory given by the Kubo formula with full frequency dependence.

Let us illustrate our results on the typical example given in Ref. 14, where a GaAs sample with hole density $n$ $=10^{19} \mathrm{~cm}^{-3}$, corresponding to a Fermi energy of the order of a few ten meV, and a mobility of $\mu=e \tau / \mathrm{m}^{*}$ $=50 \mathrm{~cm}^{2} / \mathrm{V} \mathrm{s}$. To obtain an upper bound for $\tau$ we take $m^{*}$ to be the heavy-hole mass, $m^{*} \approx 0.5 \mathrm{~m}$, corresponding to $\gamma_{1}$ $\approx 7$ and $\gamma_{2} \approx 2.5$ for GaAs. ${ }^{36}$ This leads to a lower estimate for $\hbar / \tau$ being also of the order of a few ten meV. Thus, in the above scenario, the finite momentum relaxation time $\tau$ must be taken into account when calculating the spin-Hall conductivity, differently from the approach in Ref. 14.

\section{CONCLUSIONS}

We have studied the spin-Hall transport of electrons and holes in semiconductors using the Kubo formula in the correct zero-frequency limit taking into account the finite momentum relaxation time of carriers in real semiconductors. This approach allows to analyze the range of validity of recent theoretical findings. ${ }^{14-16}$ In particular, the spin-Hall conductivity is found to vanish for vanishing spin-orbit coupling if the correct zero-frequency limit is performed. In the case of conduction band electrons in the presence of Rashba spinorbit coupling in a high-mobility quantum well, spin-orbit interaction dominates, for typical experimental for the rashba coefficient, the effects of momentum relaxation, and the spin-Hall conductivity is close to its "universal" value as predicted in Ref. 15. This situation can be different for typical $p$-doped bulk samples, where dissipation can substantially affect the spin-Hall transport.

\section{ACKNOWLEDGMENTS}

We thank J. Sinova and A. H. MacDonald for useful correspondence. This work was supported by NCCR Nanoscience, the Swiss NSF, DARPA, and ARO.
${ }^{1}$ S.A. Wolf, D.D. Awschalom, R.A. Buhrman, J.M. Daughton, S. von Molnar, M.L. Roukes, A.Y. Chtchelkanova, and D.M. Treger, Science 294, 1488 (2001).

${ }^{2}$ Semiconductor Spintronics and Quantum Computation, edited by D.D. Awschalom, D. Loss, and N. Samarth (Springer, Berlin, 2002).

${ }^{3}$ E.I. Rashba, Physica E 20, 189 (2004).

${ }^{4}$ S. Datta and B. Das, Appl. Phys. Lett. 56, 665 (1990).

${ }^{5}$ J. Schliemann, J.C. Egues, and D. Loss, Phys. Rev. Lett. 90, 146801 (2003).

${ }^{6}$ M.G. Pala, M. Governale, J. König, and U. Zülicke, cond-mat/0212560 (unpublished); M.G. Pala, M. Governale, J. König, U. Zülicke, and G. Iannaccone, Phys. Rev. B 69, 045304 (2004).

${ }^{7}$ S. Saikin, M. Shen, M.-C. Cheng, and V. Privman, J. Appl. Phys. 94, 1769 (2003); M. Shen, S. Saikin, M.-C. Cheng, and V. Privman, Lect. Notes Comput. Sci. 2668, 881 (2003); Y.V. Pershin, cond-mat/0311223 (unpublished).

${ }^{8}$ E.G. Mishchenko and B.I. Halperin, Phys. Rev. B 68,
045317 (2003)

${ }^{9}$ A. Lusakowski, J. Wrobel, and T. Dietl, Phys. Rev. B 68, 081201(R) (2003).

${ }^{10}$ R. Winkler, Phys. Rev. B 69, 045317 (2004).

${ }^{11}$ S.D. Ganichev, V.V. Bel'kov, L.E. Golub, E.L. Ivchenko, P. Schneider, S. Giglberger, J. Eroms, J. DeBoeck, G. Borghs, W. Wegscheider, D. Weiss, and W. Prettl, cond-mat/0306521 (unpublished).

${ }^{12}$ J. Schliemann and D. Loss, Phys. Rev. B 68, 165311 (2003).

${ }^{13}$ K.C. Hall, W.H. Lau, K. Gundogdu, M.E. Flatte, and T.F. Boggess, Appl. Phys. Lett. 83, 2937 (2003).

${ }^{14}$ S. Murakami, N. Nagaosa, and S.C. Zhang, Science 301, 1348 (2003).

${ }^{15}$ J. Sinova, D. Culcer, Q. Niu, N.A. Sinitsyn, T. Jungwirth, and A.H. MacDonald, cond-mat/0307663 (unpublished).

${ }^{16}$ D. Culcer, J. Sinova, N.A. Sinitsyn, T. Jungwirth, A.H. MacDonald, and Q. Niu, cond-mat/0309475 (unpublished).

${ }^{17}$ M.I. Dyakonov and V.I. Perel, Phys. Lett. A 35, 459 (1971). 
${ }^{18}$ J. Hirsch, Phys. Rev. Lett. 83, 1834 (1999).

${ }^{19}$ F. Meier and D. Loss, Phys. Rev. Lett. 90, 167204 (2003).

${ }^{20}$ T. Jungwirth, Q. Niu, and A.H. MacDonald, Phys. Rev. Lett. 88, 207208 (2002); D. Culcer, A. MacDonald, and Q. Niu, Phys. Rev. B 68, 045327 (2003), and references therein.

${ }^{21}$ E.I. Rashba, Fiz. Tverd. Tela (Leningrad) 2, 1224 (1960) [Sov. Phys. Solid State 2, 1109 (1960)]; Y.A. Bychkov and E.I. Rashba, J. Phys. C 17, 6039 (1984).

${ }^{22}$ G. Dresselhaus, Phys. Rev. 100, 580 (1955).

${ }^{23}$ G. Mahan, Many-Particle Physics, 3rd ed. (Kluwer Academic, New York, 2000).

${ }^{24}$ D. Loss, Physica A 139, 505 (1986); 139, 526 (1986); D. Loss and A. Thellung, ibid. 144, 17 (1987).

${ }^{25}$ See, e.g., H. Smith and H.H. Jensen, Transport Phenomena (Clarendon, Oxford, 1989); J.M. Ziman, Principles of the Theory of Solids (Cambridge University Press, Cambridge, 1972).

${ }^{26}$ J. Nitta, T. Akazaki, H. Takayanagi, and T. Enoki, Phys. Rev. Lett. 78, 1335 (1997).

${ }^{27}$ G. Engels, J. Lange, T. Schäpers, and H. Lüth, Phys. Rev. B 55, 1958 (1997).
${ }^{28}$ J.P. Heida, B.J. van Wees, J.J. Kuipers, T.M. Klapwijk, and G. Borghs, Phys. Rev. B 57, 11911 (1998).

${ }^{29}$ C.-M. Hu, J. Nitta, T. Akazaki, H. Takayanagai, J. Osaka, P. Pfeffer, and W. Zawadzki, Phys. Rev. B 60, 7736 (1999).

${ }^{30}$ D. Grundler, Phys. Rev. Lett. 84, 6074 (2000).

${ }^{31}$ Y. Sato, T. Kita, S. Gozu, and S. Yamada, J. Appl. Phys. 89, 8017 (2001).

${ }^{32}$ G. Lommer, F. Malcher, and U. Rössler, Phys. Rev. Lett. 60, 728 (1988).

${ }^{33}$ B. Jusserand, D. Richards, H. Peric, and B. Etienne, Phys. Rev. Lett. 69, 848 (1992).

${ }^{34}$ B. Jusserand, D. Richards, G. Allan, C. Priester, and B. Etienne, Phys. Rev. B 51, 4707 (1995).

${ }^{35}$ J.M. Luttinger, Phys. Rev. 102, 1030 (1956).

${ }^{36}$ I. Vurgaftman, J.R. Meyer, and L.R. Ram-Mohan, J. Appl. Phys. 89, 5815 (2001).

${ }^{37}$ The zeroth order in Eq. (19) agrees with the second expression given in Eq. (18) of Ref. 16 for the spin-Hall conductivity, up to a factor of $1 / 3$ which is due to different definitions of the spincurrent operator. 